\section{A cosmic rod in pickle}

SIR-Under this title, Hoyle and Wickramasinghe ${ }^{1}$ have tried to defend their various positions, and the claim that their earlier predictions had "wilfully and even maliciously been overlooked".

It seems clear, however, that the real reason that their work is not widely accepted or referred to is not that they have "sinned mortally" by making a "contact between biology and astronomy", but because so many of their various claims, put forward with so much certainty on the basis of unimpressive and even inaccurate evidence, have routinely been abandoned by themselves or proved wrong by others (see refs 2 and 3 ). Indeed, even before publication, their Nature article had been rendered obsolete by a published paper ${ }^{4}$ they had submitted on 29 November 1985 , but did not refer to. This paper claimed hollow, spherical $\mathrm{C}_{60}$ (buckminsterfullerene) molecules' as the explanation of the composition and ultraviolet (UV) spectrum of interstellar grains that had been earlier identified, inter alia, as dried hollow bacteria ${ }^{6,7}$. If the absorbence in the infrared (IR) were really due to such bacteria the absorbence in the UV would be far greater than in the $I^{8.9}$. They cannot have it both ways, especially as the UV and IR spectra of this $\mathrm{C}_{60}$ molecule have not yet been measured (R.F. Curl, personal communication).

But there are other problems. As it was part of their Fig. 1, Karim, Hoyle and Wickramasinghe had to have known that the rest of the absorption spectrum of tryptophan did not fit the curves in their Figs 3 and 5 when they claimed that "the discovery of a broad interstellar absorption feature centred on the $\lambda 2,800 \AA$ in the extinction curve of starlight confirms the presence of proteinaceous material in grains" 10 . In ref. 12, they must have deliberately cut off the low wavelength part of their own (mislabelled) curve in Fig. 1 to make it fit the curves in Fig. 4 to support one of their many now abandoned theories. (For further comment, see refs 2-4, 8 and 11.) Also, Hoyle et al. ${ }^{6}$ knew that my colleagues and $I^{2}$ had never claimed the existence of a peak for Escherichia coli at $220 \mathrm{~nm}$, and so on. This type of scientific behaviour is clearly unacceptable.

In the past few years, many comments on their publications have appeared. For example, Greenberg ${ }^{13}$, McLachlan and Nandy $^{14.15}$ and Savage and Sitko ${ }^{16}$ have shown that the claimed ${ }^{10}$ absorption feature at $\lambda=2,800 \AA$ was produced by saturation effects in overexposed spectra and was flagged as such in the data bank.

Thus, the long history of unwarranted claims by Hoyle and Wickramasinghe means that I, and many other scientists, will not accept anything they claim, be it about darwinism, viruses, bacteria, Archaeopteryx, or even interstellar grains or steady-state cosmology, unless and until it is demonstrated by others with far better batting averages and about whose works it cannot be said "That's not cricket". By the way, astronomers and chemists, also, have "rods in pickle"* for them too.

R.E. Davies

Departments of Animal Biology, and Astronomy and Astrophysics,

University of Pennsylvania,

Philadelphia, Pennsylvania, 19104, USA

1. Hoyle, F. \& Wickramasinghe, N.C. Nature, 332, 509-511 (1986)

2. Davies, R.E., Delluva, A.M. \& Koch, R.H. Nature 311, $748-750(1984)$

3. Davies, R.E. Panspermia: Unlikely, Unsupported, but Just Possible. Preprint 36th Congress of the International Astronautical Federation. Stockholm, Sweden. Octobe 7-12 (1985). Int. Astro. Fed. (Pergamon, Oxford).

4. Hoyle, F \& Wickramasinghe, N.C. Astrophys. Space Sci. 122, 181-184 (1986)

5. Kroto, H.W., Heath, J.R., O'Brien, S.C., Curl, R.F. \& Smalley, R.E. Nature 318, 162-163 (1985).

6. Hoyle, F., Wickramasinghe, N.C. \& Al-Mufti, S. Astro phys. Space Sci. 98, 343-352 (1984).

7. Hoyle, F., Wickramasinghe, N.C. \& Al-Mufti, S. Astrophys. Space Sci. 110, 401-404 (1985)

8. Davies, R.E., Delluva, A.M. \& Koch, R.H. in The Search for Extra-terrestrial Life - Recent Developments Int. astr. Un. symp. 112 (ed. Papagiannis, M.D.) 165-169 (Reidel Dordrecht, 1985

9. Yabushita, S., Wada, K., Takai, T., Inagaki, T., Young, D. \& Arakawa, E.T. Astrophys. Space Sci. 124, 377-388 (1986).

10. Karim, I.M., Hoyle, F. \& Wickramasinghe, N.C. Astro phys. Space Sci. 94, 223-229 (1983)

11. Koch, R.H. \& Davies, R.E. Astrophys. Space Sci. 100 $425-426(1984)$

12. Karim, L.M., Hoyle, F. \& Wickramasinghe, N.C. Astro phys. Space Sci. 100, 431-435 (1984)

13. Greenberg, J.M. Observatory 104, 134-135 (1984)

14. McLachlan, A. \& Nandy, K. Observatory 104, 29-31 (1984).

15. McLachlan, A. \& Nandy,K. Astrophys. Space Sci. 109 399-402 (1985)

16. Savage, B.D. \& Sitko, M.L. Astrophys. Space Sci. 100 $427-429(1984)$

19. Partridge, E. A Dictionary of Slang and Unconventional English 7th edn (Macmillan, New York, 1972)

*For those not familiar with this somewhat obscure term, a "rod in pickle", which Hoyle claims to have "not a few of", does no mean a venereally infected penis (UK), a good horse that is being either reserved or nursed for a sure win (Australia), pistol in a plight (USA), but a prospective punish (Australia), ing (UK) 18

\section{Indian collaboration}

SiR-The news item "Indian science: Foreign software giants pounce" (Nature 323, 102; 1986) states "Astra's subsidiary in Bangalore has taken the cream of Indian biochemists, including the chief biotechnologist of the Indian Institute of Science". As I am a biochemist who has acquired the label "biotechnologist" and am intimately involved in the organization of the Astra centre, I wish to say that the statement is not correct. I am not on the payroll of Astra nor have any of my colleagues left the institute to join Astra. The core group at the centre consists of fresh $\mathrm{PhDs}$ who were ready to leave the country and take up postdoctoral fellowships in the United States. Besides, Astra is trying to bring back qualified Indians from abroad, so the centre is helping to prevent a brain-drain, both internal and external.

Astra has given funds to the Indian Institute of Science, enabling me to further my basic research, which I have been carrying out for several years. At the same time, we have an opportunity to interact with the centre on problems related to the alleviation of human suffering. The Astra centre seems to us to be a unique, open and fair collaboration between an academic and an industrial research institution. The Astra centre in Bangalore is not a subsidiary of Astra Sweden. The centre will develop its own know-how and is recognized by the government as a non-profit research organization. It is registered as a society in Karnataka State. The government of India has laid down conditions to protect the Indian interests in terms of publications, patents, royalties and the conduct of research.

There is much talk about the virtues of collaboration between industry and academic research. There is also criticism that scientists in India have not been doing relevant research and that they should have discovered cures for tropical diseases a long time ago. Now that scientists are trying out a new model of collaboration and when the government has done what it can to protect Indian interests, it is a pity to lump together and to condemn all multinational efforts. I wish K.S. Jayaraman had made a deeper study of this collaboration model before offering his comments or those of his critics, which could harm not only the institution but the entire philosophy.

G. Padmanaban

Department of Biochemistry,

Indian Institute of Science, Bangalore 560 012, India

\section{Faraway quasars}

Sir-You quote me (Nature 323, 193; 1986) as saying that it is only a matter of time before very long baseline interferometry yields the absolute distances to at least one quasar with an interposing gravitational lens. Alas, were that only true. What I said, in fact, was that we know of no way to determine directly the distances to quasars. But if a gravitational lens between the quasar and us forms multiple images, then, if the quasar cooperates, for example by changing its luminosity sharply, it is possible, at least in principle, to determine the differences in the times taken by light to propagate from the quasar to us along the paths for the different images. I stated my opinion that it would just be a matter of time before an imaged quasar cooperated sufficiently for us to determine such time differences.

Harvard-Smithsonian Center IRWIN SHapiro

for Astrophysics,

60 Garden Street

Cambridge, Massachusetts 02138, USA 